\title{
Middle Ear Inflammation, CTCAE
}

National Cancer Institute

\section{Source}

National Cancer Institute. Middle Ear Inflammation, CTCAE. NCI Thesaurus. Code C143673.

A disorder characterized by inflammation (physiologic response to irritation), swelling and redness to the middle ear. 